\section{Caracterización económica de la mujer rural'}

\author{
Economic characterization of rural women
}

\section{Ana Hercilia Hamón Naranjo*}

Recibido: 29-08-2014 / Revisado: 29-09-2014 / Aceptado: 09-05-2015

\section{Resumen}

El objetivo de esta investigación es caracterizar económicamente a las mujeres rurales, para visibilizar, valorar y resaltar el gran aporte que hacen al desarrollo rural, donde ellas como protagonistas fundamentales realizan diferentes actividades económicas dentro y fuera del hogar, con el fin de lograr un incremento de sus ingresos para alcanzar un crecimiento en algunos casos y en otros para sobrevivir ellas y sus familias.

En esta investigación se abordaron los siguientes temas: actividad productiva, remuneración de sus labores, propiedades económicas, administración del dinero producto de la actividad económica y participación en la actividad comercial. El resultado permite demostrar la importancia del trabajo de las mujeres rurales, pero que no se reconoce, ni valora y, por consiguiente, no se visibiliza. Es un reto adoptar los cambios que se

\footnotetext{
1 Artículo de reflexión, producto de la investigación para la tesis de Maestría de Desarrollo Rural de la Universidad Pedagógica y Tecnológica de Colombia La mujer rural dentro del contexto de desarrollo rural. Un análisis para el municipio de Santa Sofía, Boyacá.

* Doctora en Ciencias de la Educación. Magíster en Desarrollo Rural. Especialización en Alta Gerencia en Mercados, Especialización en Finanzas. Administradora de Empresas. Asesora Gobernación de Boyacá. Docente universitaria de posgrados y pregrado de la Universidad de Boyacá y la Universidad Pedagógica y Tecnológica de Colombia. Integrante del Grupo de Investigación Historia y Prospectiva de la Universidad Latinoamericana HISULA, Universidad Pedagógica y Tecnológica de Colombia. anahamon20@yahoo.es
}

presentan en el mundo para mejorar su situación y, por ende, el de la sociedad en general.

Se utilizó la investigación cualitativa y cuantitativa, con el propósito de ampliar la capacidad para captar información, analizar e interpretar la realidad económica. Como unidad de estudio y trabajo se estableció un grupo conformado por 30 mujeres rurales, mayores de 18 años, sin importar el estado civil, la actividad económica, el nivel educativo o la condición social, pero que estén radicadas en el municipio de Santa Sofía, Boyacá.

Palabras clave: mujer, economía, desarrollo rural. 


\section{Citación}

Hamón, A. (2015). Caracterización económica de la mujer rural. Visión Empresarial, 1 (1). 88-100.

\section{Abstract}

The objective of this research is to characterize economically the rural women, to make visible and evaluate their important contribution to rural development, where they as fundamental actors perform different economic activities inside and outside the home, in order to achieve an increase of their income to get a growth in some cases and in others to survive them and their families.

In this research the following topics were addressed: productive activity, remuneration of their work, properties, money management product of economic activity and participation in business. The result allows demonstrate the importance of the work of rural women, but it is not recognized and thus not visible. It is a challenge to adopt the changes that occur in the world to improve their situation and therefore society in general.

Qualitative and quantitative research was used, in order to get information and analyze economic reality. The population of this research is a group of 30 rural women, 18 years or older, regardless of marital status, economic activity and education level or social status, but which are located in rural area of Santa Sofia, Boyacá.

Keywords: women, economy, rural development.

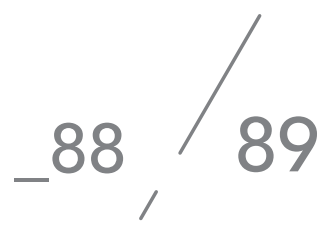

\section{Introducción}

El objetivo de la presente investigación es identificar y caracterizar el papel de la mujer rural del municipio de Santa Sofía, Boyacá, dentro del contexto del desarrollo rural en el aspecto económico, para resaltar, valorar y visibilizar su trabajo, mediante el análisis de los siguientes temas: actividad productiva, remuneración de sus labores, propiedades económicas, administración del dinero producto de la actividad económica y participación en la actividad comercial.

Esta investigación es de gran importancia porque permite reconocer el triple rol de las mujeres rurales, como reproductoras, trabajadoras domésticas y trabajadoras productivas. Adicionalmente visibiliza la 
significativa función de la mujer en la economía, puesto que a través de la producción y venta de servicios genera ingresos adicionales a la familia, a pesar de la falta de valoración de la mayoría de las actividades que ella realiza, aspecto preocupante y que será necesario estudiarlo en otra investigación.

Metodológicamente se utilizó la investigación cualitativa con la cuantitativa. Las técnicas fueron: la encuesta, la observación participante, el diario de campo, historias de vida y taller, las cuales fueron escogidas por su correspondencia con los métodos empleados en esta investigación, por ser adecuadas para la comprensión de la realidad presentada, con el fin de ampliar la búsqueda de información y conocer su aporte a la economía y al desarrollo rural.

\section{Caracterización económica de la mujer rural}

\section{Actividad productiva de la mujer rural}

La mujer rural del municipio de Santa Sofía desarrolla una importante y variada labor productiva que, a su vez, representa un aporte significativo al desarrollo rural. En primer lugar, el trabajo doméstico; en segundo lugar, la producción agropecuaria con destino al autoconsumo y una pequeña parte para el mercado local, y en tercer lugar, el trabajo de venta de su fuerza laboral extrapredial.
El trabajo productivo se refiere a la actividad agrícola realizada en la huerta casera o "de pan coger", en donde se cultivan varios productos en forma simultánea para el autoconsumo, lo que se constituye en la base de la seguridad alimentaria para la familia. Los productos cultivados son hortalizas, frutas, verduras, aromáticas, maíz y papa, entre otros; igualmente, cuidan algunos animales como aves, cerdos, ovejas, vacas y conejos.

Cuando realiza trabajo extrapredial, lo hace en casas de familia, en labores domésticas, en la escuela como ecónoma, y en actividades agrícolas, especialmente en recolección, selección y empaque; en algunos casos también como administradora de cultivos de tomate de invernadero y uchuva.

Existe gran controversia sobre el trabajo doméstico de la mujer, puesto que las instituciones del Gobierno nacional encargadas de tomar decisiones fundamentales, consideran que el trabajo el doméstico es improductivo, que no aporta nada a la economía, cuando en realidad este trabajo es la base de la economía campesina y del desarrollo rural.

Este trabajo doméstico no se puede desconocer, ya que es importante porque:

(...) desde el punto de vista de la producción doméstica, al campesinado se le ha caracterizado por la generación de productos para 
su subsistencia o para el mercado, realizado por trabajadores asalariados, arrendatarios o propietarios $\circ$ por productores que transitan por estas posiciones de manera temporal e independiente. Por producción doméstica se entiende la organización de la producción realizada, al menos parcialmente, a través de las relaciones de poder y autoridad existentes dentro del hogar o también por relaciones de parentesco y la ausencia de cualquier organización burocrática o impersonal. (Salgado \& Prada, 2000, p.39).

La contribución de la mujer a la actividad productiva se describe en la información obtenida mediante los diferentes instrumentos. Por ejemplo, un entrevistado manifestó que las mujeres cuidan:

Gallinas, conejos, ovejas, piscos; cocinan a obreros; hacen oficios caseros; trabajan en las huertas; cultivos con el esposo; hilan lana para cobijas y ruanas, sacan fique para hacer lazos para el uso de la casa o para vender en el mercado del pueblo. (Relato 21).

En el trabajo extrapredial la mujer rural también realiza una actividad importante:

Cuando no hay trabajo en la casa salgo a donde los vecinos a ordeñar y apartar los terneros: le dan $\$ 20.000$, diarios y una botella de leche y gasta esta platica para el mercado; además de trabajar en la casa en todos los oficios varios, va al pueblo a trabajar en casas de familia, donde le pagan \$15.000, por día. (Relato 12 ).

El trabajo de la mujer es intenso; a veces sale a trabajar a otras fincas, cocina, lavar ropa, también prepara la cuajada para el queso y hace pan para vender el domingo en el pueblo, como relatan algunas de ellas:

Salgo a la escuela a trabajar como ecónoma, hasta la 1 ○ 2 p.m. me pagan 100.000 mensuales, que los invierto en alimentación, ropa para mis hijos y lo que necesite. (Relato 9).

Además de trabajar en la casa en todos los oficios varios, va al pueblo a trabajar en casas de familia, donde le pagan $\$ 15.000$ por día. (Relato 13 ).

Lo anterior ratifica el papel económico y social que realizan hombres y mujeres en pequeñas unidades de producción agropecuarias:

Allí queda claro que son las mujeres las que cumplen principalmente el triple rol de reproductoras, trabajadoras domésticas y trabajadoras productivas. Esto significa que la mayor parte de las mujeres campesinas dedica a diario unas horas a la manutención de su familia a través del cuidado de niños y niñas, la preparación de alimentos, el cuidado de la huerta familiar, el lavado de la ropa, entre otras; además, participa en las tareas productivas de preparación 
de tierras, cosecha y cuidado de animales, e incluso en el mercado de los productos; sin embargo, su contribución es mayor en el trabajo doméstico de cultivos pequeños de productos para el mercado, y de transformación de alimentos. (Ospina, 1992, p.13).

Lo anterior hace pensar que:

para entender el papel que las mujeres rurales juegan en la seguridad alimentaria, es necesario comprender ésta no sólo como la producción y comercialización de alimentos para la satisfacción de las necesidades básicas de poblaciones dadas, sino en términos de la sostenibilidad de esa población en el tiempo, y la participación en los procesos de diversificación del trabajo rural. (Ospina, 1992, p.13).

La mujer rural dentro del contexto de género es participante activa en los procesos productivos y dueña de una serie de actividades que contribuyen al desarrollo rural dentro del ámbito de la sostenibilidad ambiental y económica, haciendo de sus labores una oportunidad para demostrar el verdadero papel que desempeña en la familia y en la sociedad como soporte de unidad y como integrante de un grupo productivo.

\section{Remuneración de la labor realizada por la mujer rural}

El $97 \%$ de la mujeres no recibe remuneración por las actividades que realiza en el hogar, por las razones antes descritas; solo el 3 \% obtiene algún ingreso, y corresponde a quienes realizan actividades en el sector agrícola, de producción y comercialización o laboran en el restaurante escolar; se caracterizan porque desarrollan el trabajo fuera de la casa, reciben baja remuneración y pertenecen al grupo de estado civil solteras o separadas.

De acuerdo con lo expresado, el total de las mujeres que desarrollan actividades dentro del hogar no recibe remuneración alguna por su trabajo, lo reciben solo quienes laboran extrapredial, es decir, en lugares diferentes a su hogar.

El hecho de que la mujer rural no reciba reconocimiento por las actividades que realiza dentro del hogar no quiere decir que sea una generadora pasiva de ingresos, porque algunas actividades y trabajos extraprediales le reportan dinero que gasta en el sustento de la familia, como se describe en los siguientes relatos:

De la leche se produce cuajada para vender los domingos, aproximadamente 9 libras por semana, cada una a \$1.100; a veces no hay ninguna producción por no existir vacas con cría y, por consiguiente, se vuelve difícil la situación, la pla- 
ta de la cuajada es para comprar la cebolla, el tomate y algo de tienda. (Relato 18).

Salgo a trabajar tres días a la semana en un restaurante donde me pagan $\$ 20.000$ por día; a veces voy a lavar, planchar o a hacer de comer a hacer aseo y me pagan $\$ 15.000$ de 8 am a 4 pm. (Relato 15).

Es decir, que en promedio recibe por semana $\$ 12.500$, que en un mes asciende a un total de $\$ 50.000$, cifra muy distante del mínimo establecido para el sector rural.

Con la familia corto y saco fique para hacer lazos para el ganado que se tiene, y para vender el día domingo en la plaza de mercado; se hacen entre 10 y 15 lazos semanales que se venden entre $\$ 1.000$ y $\$ 1.500$; con esta platica se hace el mercado para la semana. También se cuidan ovejas para sacar la lana para las cobijas y ruanas para el grupo familiar, que también se venden. En promedio se reciben $\$ 15.000$ semanalmente. (Relato 2).

También se cuidan vacas para la producción de leche y sacar quesos, 70 cada domingo para la venta en el pueblo, cada uno vale $\$ 1.000$, o sea un total de $\$ 70.000$ semanal para el mercado de la casa y los gastos que se necesiten, esporádicamente saca un tiempo para tejer cubrelechos en hilo para la casa. (Relato 3).
En mi trabajo yo cuido pollos, vacas, vendo el $70 \%$ de la leche al carro que la recoge todos los días y la lleva para Moniquirá al Trópico, la pagan a $\$ 320$ la botella; semanalmente me entregan \$15.700; también cuido las ovejas, cerdos, aves, los huevos los vende ella cada uno a $\$ 300$, y con esa platica hace parte de mercado de tienda. (Relato 19).

Teniendo en cuenta los relatos de la mujeres rurales, son pocas las actividades que se le remuneran; se encontró que solo cuando van a trabajar fuera de su casa, como ya se mencionó, reciben algo por su labor, por ejemplo en restaurantes escolares ganan $\$ 100.000$ mensuales, restaurantes del centro del pueblo $\$ 20.000$, día, y por lavar, planchar, asear y cocinar en casas de familia les pagan \$15.000 día. Valores que demuestran lo planteado respecto a la subvaloración del trabajo realizado por las mujeres.

Esto indica que la remuneración de las actividades de la mujer, en primer lugar, es baja, y en segundo lugar, son pocas las actividades remuneradas; además, lo que reciben por su trabajo está por debajo de todos los parámetros legales y, lo más grave, limita toda posibilidad de progreso, por no tener recursos o medios para mejorar su nivel de vida.

Como lo referencia León (1982a), en todos los casos, el trabajo doméstico no es remunerado, lo hace la mujer, y consiste en una producción no mercantil. Sin embargo, 
existen diferencias en la forma como cada producción doméstica se vincula al mercado y en la cantidad y tipo de producción que se lleva a cabo. Los nexos con el mercado son más directos en el caso del contexto agrícola, en el cual los insumos para la producción doméstica no se compran en mercado, o cuando la fuerza de trabajo no se vende por un salario.

Al analizar lo expuesto se puede considerar que es necesario el reconocimiento y contabilización del aporte económico de las mujeres a la producción de la fuerza de trabajo (León, 1982a) y al abastecimiento alimentario, porque, como se ha afirmado, son muchas las actividades que realizan y poca la retribución social y económica que reciben, lo cual hace que se presente inequidad en el trato, no solo como género sino también como integrante de una sociedad en la cual interactúa activamente.

La mujer como integrante de una sociedad campesina cumple varios roles, produce para la subsistencia, produce para otros sectores alimentos y materias primas, produce ingresos para sí misma y para el grupo familiar (Salgado, 2000, p.60), sin que reciba una remuneración justa y equitativa que le permita acceder a un mejor nivel de vida en buenas condiciones de salud, educación, vivienda y servicios públicos.

\section{Propiedades económicas de la mujer}

El $60 \%$ de las mujeres encuestadas no posee propiedades de ninguna naturaleza, el $26 \%$ tiene animales, entre los que se encuentran especies menores (gallinas, conejos, ovejas y cerdos) y vacunos; el $14 \%$ de las mujeres es dueña de predios que en su mayoría han sido recibidos por herencia y son explotados por el marido.

Respecto a la propiedad, es necesario tener en cuenta que aunque la mujer figure como propietaria, la explotación económica la lleva a cabo el esposo, es decir, que quien hace producir la tierra a través de los cultivos y obtiene para sí los ingresos, no es el dueño, y quien posee los títulos no recibe beneficio alguno diferente a los medios de subsistencia, por el hecho de ser pareja. Respecto a las especies menores y vacunos la situación es diferente, con el agravante de que la mujer es quien está a cargo de su cuidado.

Dentro de este contexto, es importante tener en cuenta que el acceso de las mujeres a la tierra se puede constituir en un factor importante de empoderamiento, en torno al cual las mujeres pueden convertirse en ciudadanas de primer nivel. Se ha demostrado que los derechos formales de la mujer sobre la tierra influyen en el poder de negociación que tiene en el hogar y en la comunidad (Ospina, 1999, p.32). 
La propiedad y tenencia de la tierra se convierten en un factor de igualdad social, toda vez que le permite a la mujer asumir actitudes propias frente a la subsistencia, relación de pareja y toma de decisiones respecto al quehacer diario y la adopción de formas de vida que beneficien su vejez.

\section{Administración del dinero producto de la actividad económica de la mujer rural}

Del reducido porcentaje de mujeres que reciben remuneración por la actividad que realizan, el $56 \%$ tiene autonomía para su manejo, y decide cómo gastarlo, invertirlo o ahorrarlo, que corresponde al grupo constituido por las mujeres solteras o separadas, en su mayoría, y el $44 \%$ debe destinar sus ingresos para el sustento familiar y, en consecuencia, la administración del dinero no la realiza a voluntad propia, sino que obedece a los requerimientos del grupo familiar.

Lo anterior hace pensar que la situación de la mujer respecto a la administración del dinero no es la mejor, porque, en la mayoría de los casos, no recibe ingresos por la actividad que realiza, y quienes sí lo reciben, el $44 \%$, no tiene autonomía para su manejo, pues deben obedecer a otras personas.

En el manejo del dinero, la mujer en muchas oportunidades es considerada como una menor de edad, sin responsabilidad para dicho manejo, por lo cual no es extraño ver como los señores son los que reciben todo el dinero y deciden como gastarlo, incluso el generado por las mujeres, producto del cuidado de especies menores de venta de productos lácteos. En el mercado, la señora debe pedir al esposo la plata para pagar las compras, lo que obliga a que dentro de su comportamiento sumiso deba aceptar la cantidad que le ofrece el hombre.

Sin embargo, algunas mujeres administran sus ingresos, se muestran seguras, confiadas e independientes, dispuestas a tomar sus propias decisiones respecto a las compras y, en general, a la inversión del dinero; lo que hace pensar que el manejo del dinero es un determinante de poder dentro de la sociedad rural en la cual se encuentra inmersa.

\section{Valoración del trabajo de la mujer rural}

Como conclusión del análisis de encuestas, talleres, relatos y hojas de vida, el 86 \% de las mujeres rurales considera que su trabajo no es valorado, y corresponde a quienes no reciben ingresos y realizan actividades domésticas; el $14 \%$ manifiesta que sí lo reciben, y pertenece a aquellas que desarrollan labor extrapredial; dicha remuneración es baja y no responde a lo establecido por el Gobierno como salario mínimo para el sector rural.

Se considera que el ingreso que recibe la mujer trabajadora en el área de estudio es bajo, porque no alcanza al mínimo establecido legalmente, no incluye 
prestaciones ni pago al sistema general de seguridad social, los horarios son superiores a las 8 horas y los riesgos de enfermedades son elevados por la falta de programas de seguridad industrial.

Aunque en la forma pura de economía campesina hay diferencias entre hombres y mujeres, lo más importante para entender la situación de la mujer en el área rural son las relaciones de dominación y las nuevas modalidades que asume con la dominación del capitalismo a la agricultura y la creciente urbanización. Si bien persisten relaciones sociales de producción no capitalistas que generan formas de dominación extraeconómicas y que adquieren gran importancia en la división sexual del trabajo (León, 1982b, p. 41).

Hablar de la baja valoración del trabajo de la mujer es volver a lo ya expresado; por tanto, se considera pertinente asumir una actitud más crítica respecto a todo el contexto del trabajo, para que en la propuesta final de lineamientos se presente un mayor acercamiento a la realidad vivida por la mujer campesina y a la necesidad de empoderamiento, a fin de poder alcanzar mejores condiciones de vida dentro de la economía campesina imperante.

Una de las características sobresalientes de la economía campesina radica en que la familia es, al mismo tiempo, unidad de producción y unidad central de reproducción de la fuerza de trabajo familiar. Por lo tanto, no hay gran desvinculación entre las actividades de producción directa, como la agricultura, y la producción de bienes requeridos para el mantenimiento diario de la familia (León, 1982b, p.19).

\section{Participación de la mujer en la actividad comercial}

Un tema que se debe examinar dentro de la actividad económica y productiva de la mujer rural del área de estudio, es su participación en el mercado como oferente, intermediaria o demandante, ya que se caracteriza por ser muy activa en la compra de productos para el sustento de hogar y de su actividad productiva, como alimentos, vestuario e insumos agrícolas, principalmente, y en la venta de productos agropecuarios generados en la actividad económica de la familia:

Entre los que se encuentran huevos, leche, cu jada, queso, mantequilla, lazos, lana y frutas, entre otros; los cuales son comercializados generalmente los domingos en las plazas de mercado del municipio y de la región (Relato 7).

Las mujeres rurales llegan al centro del pueblo, directamente a la plaza de mercado; algunas lo hacen en camperos, otras, en camión y unas pocas, a pie; llegan bien vestiditas, limpias, la mayoría con su sombrero, ruana y un canasto donde llevan lo que van a vender como huevos, cuajadas, mantequilla, frutas, lazos y gallinas. A veces las acompaña el esposo o algunos de sus hijos pequeñitos que siempre los llevan al lado; 
además, el niño les ayuda, y la mamá les enseña a vender los productos.

Cuando son pequeñas cantidades, también algunas llevan costales pequeños donde empacan gallinas, pollos, lazos, lana para vender al detal a gente del pueblo o a personas que vienen al mercado de otros pueblos como Villa de Leyva, Sutamarchán, Tunja y Bogotá, entre otros.

La asistencia al mercado es aprovechada para saludar a los familiares o vecinos $y$, algunas veces, para que las contraten para trabajar en otras fincas, o para conseguir obreros para el trabajo de sus fincas, o realizar alguna otra transacción.

Luego de vender sus productos se van a misa de 10 de la mañana o de 12; después van a hacer el mercado de tienda, si les alcanza la plata que le pagaron por los productos. A veces se toman una cerveza con el esposo y algunos parientes o vecinos, dialogan y después regresan a la casa.

Las que no tienen muchos recursos salen de misa y se van para la casa; es costumbre también los domingos ir a pagar una misa por un familiar muy especial; el valor de las misas es aproximadamente de $\$ 30.000$; les gusta acompañar a los amigos y vecinos, principalmente los sábados y domingos a las misas que pagan; les ofrecen cerveza y dicen que esa compañía se devuelve en cualquier momento cuando ellas lo necesiten. (Relato 7)

En general, las mujeres rurales

además del trabajo en el escenarios doméstico, están efectiva y eficientemente vinculadas a la economía de mercado desde las etapas de producción y transformación de alimentos, pasando por la comercialización de producto y/o (sic) vinculación al mercado laboral extra predial, hasta el consumo de todo tipo de bienes. (Ospina, 1999, p.30)

Vinculación que es permanente por su rol dentro del intercambio como agentes que interactúan como productoras o consumidoras especialmente, sin dejar de lado la intermediación.

\section{Participación de la mujer en el sector financiero}

De acuerdo con la información de la gerencia de la única entidad bancaria existente en el municipio, que es el Banco Agrario, las mujeres hacen uso de los servicios de ahorro y crédito; igualmente, se estableció que existen programas directamente para ellas, con una línea de crédito llamada "Mujer rural de bajos ingresos", destinado a mujeres cabeza de familia cuyos activos totales no superen los 29 millones de pesos. 
En este tipo de crédito, el banco presta el $100 \%$ de los costos directos hasta un máximo de 29 millones, DTF + 2.E.A., con una garantía del Fondo Agropecuario de Garantías. También presta para créditos, por una cuantía de 15 salarios mínimos mensuales vigentes hasta un $100 \%$ del valor de los costos directos; otro crédito que otorga es $90 \%$ del valor, dentro de un rango de 5 a 29 millones. En la actualidad ninguna mujer rural ha solicitado esta línea de crédito, porque les da miedo endeudarse: en algunos casos, por el riesgo de las hipotecas, en otros, porque no tienen las garantías, y también, porque el marido no les permite hacer uso del crédito; además se observó que no había mucho interés por parte del Banco para promocionar esta línea de crédito, que es una buena oportunidad para las mujeres rurales.

En cuanto a ahorros, en el Banco existe un total de 359 cuentas pertenecientes a hombres y mujeres, de las cuales 106 pertenecen a mujeres, que representan el $29.5 \%$, aunque con cuantías pequeñas que oscilan entre $\$ 200.000$ y $\$ 1.000 .000$; algunas pocas tienen antigüedad superior a un año.

Con respecto al crédito, el Banco Agrario tiene vigente un total de 217 créditos, de los cuales 58 corresponden a mujeres rurales $(29.7 \%)$, con destino a microcréditos para bovinos, caña panelera, cítricos, libre inversión, cultivo de curuba, sistemas de riego y construcción de represas o reservorios; estos créditos son concedidos a plazos de 3 a 5 años, la mayoría en cuantías que oscilan entre $\$ 2.000 .000$ y $\$ 10.000 .000$.

Al respecto, es necesario explicar que:

(...) existen trabas de tipo administrativo y normativo frente al crédito ya que, como se mencionó, las mujeres no tienen título de propiedad; sin embargo, no se utilizan garantías alternativas como la prenda agraria o la firma solidaria o las certificaciones de compra de cosecha, que están contempladas en normatividad (Ospina, 1999, p.32).

Adicionalmente, la información que se posee no es muy amplia, por lo tanto, se desconocen las bondades de la financiación.

\section{Conclusiones}

Las conclusiones podrían ser bastantes, ante la riqueza económica que acompaña la vida de las mujeres rurales del municipio de Santa Sofía, Boyacá; pero es necesario resaltar las más importantes que permitan crear una imagen real de la mujer campesina de esta localidad, que es casi similar a la realidad departamental, nacional e internacional. Entre las más relevantes, tenemos:

La realidad muestra un subdesarrollo rural que excluye a la mujer de toda posibilidad de crecimiento y desarrollo económico, que la enmarca en un panorama muy crítico 
y desolador donde predomina exceso o sobrecarga de trabajo, el modelo económico y subvaloración del mismo, que invisibiliza el aporte del mujer al desarrollo rural. La falta de valoración del trabajo de la mujer, la hace más pobre. Esto se agudiza con el deterioro de la producción, la desagrarización, los cambios en las modalidades de contratación, y la extensión de las jornadas de trabajo con ingresos precarios.

Por una parte, al expresar lo anterior en cifras, se evidencia que el $60 \%$ de las mujeres encuestadas no posee propiedades de ninguna naturaleza, el $26 \%$ tiene animales, el $14 \%$ posee predios que, en su mayoría, han sido recibidos por herencia, y son explotados por el marido. Del reducido porcentaje de mujeres que reciben remuneración por la actividad que realizan, el $56 \%$ tiene autonomía para su manejo, y decide cómo gastarlo, invertirlo o ahorrarlo, que corresponde al grupo constituido por las mujeres solteras o separadas, en su mayoría, y el $44 \%$ debe destinar sus ingresos para el sustento familiar $y$, en consecuencia, la administración del dinero no la realiza por voluntad propia, sino que obedece a los requerimientos del grupo familiar. El 86 $\%$ de las mujeres rurales considera que su trabajo no es valorado, y corresponde a quienes no reciben ingresos y realizan actividades domésticas; el $14 \%$ manifiesta que sí lo reciben, y pertenece a aquellas que desarrollan labor extrapredial; dicha remuneración es baja y no responde a lo establecido por el Gobierno como salario mínimo para el sector rural.
Por otra parte, a lo largo de la investigación se demuestra el aporte significativo de la mujer como integrante de una sociedad campesina, que cumple varios roles, produce para la subsistencia, produce para otros sectores alimentos y materias primas, produce ingresos para sí misma y para el grupo familiar (Salgado, 2000, p. 60), sin que reciba una remuneración justa y equitativa que le permita acceder a un mejor nivel de vida en buenas condiciones de salud, educación, vivienda y servicios públicos.

Finalmente y ante la realidad encontrada y descrita en esta investigación, el reto para las comunidades rurales y para la sociedad en general, es adoptar los cambios que se están presentando en el mundo, fortaleciendo las acciones positivas de cada país, de manera que sea una meta el mejoramiento de las condiciones económicas de las mujeres rurales. En otros términos, valorar, reconocer y visibilizar su trabajo, para mejorar sus condiciones de vida y por consiguiente de la sociedad en general.

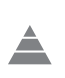




\section{Referencias}

León, M. (1982a). La realidad colombiana. Debate sobre la mujer en América Latina y el Caribe. Bogotá: ACEP Asociación Colombiana para el Estudio de la Población.

León, M. (1982b). Sociedad y subordinación. Bogotá: ACEP Asociación Colombiana para el Estudio de la Población.

Ospina, R. I. (1999). Para empoderar a las mujeres rurales. Misión Rural vol. 8. Bogotá: Tercer Mundo.

Salgado, C. \& Prada, E. (2000). Campesinado y protesta social en Colombia. Bogotá: CINEP. 